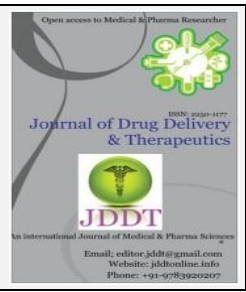

Open $\odot$ Access

Research Article

\title{
A Benign Synthesis of 2-Amino-3-Cyano-4H-Benzopyrans via Domino Reaction
}

\section{S. Silambarasan *a, A. Jamal Abdul Nasser a}

a Department of Chemistry, Jamal Mohamed College, Tiruchirappalli 620020, Tamil Nadu, India.

\section{ABSTRACT}

A green and operationally simple approach domino reaction has been developed for the synthesis of 2-amino-3-cyano- 4H-chromene derivatives from aromatic aldehydes, resorcinol and malononitrile in aqueous medium. This work represents the first example of catalyst free and organic solvents free multi-component reactions for the synthesis of pharmaceutically important chromene derivatives. The structures of the synthesized compounds were confirmed by ${ }^{1} \mathrm{H}$ NMR, ${ }^{13} \mathrm{C}$ NMR, IR, Mass and single crystal XRD.
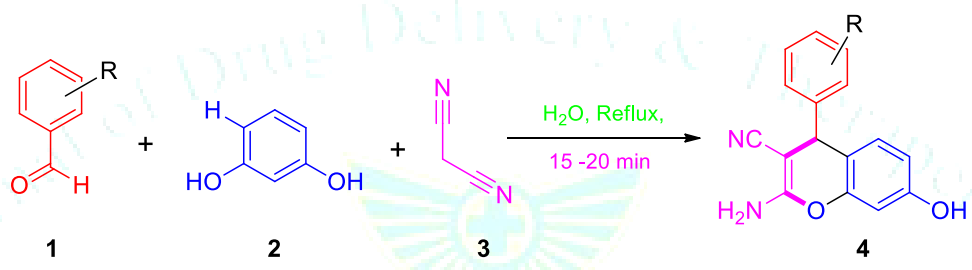

Keywords: Benzopyrans, malononitrile, Michael addition reaction, domino reaction, water mediated synthesis.

Article Info: Received 04 July 2019; Review Completed 23 Aug 2019; Accepted 26 Aug 2019; Available online 30 Aug 2019

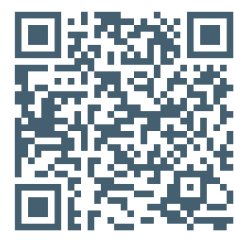

Cite this article as:

Silambarasan S, Abdul Nasser AJ, A Benign Synthesis of 2-Amino-3-Cyano-4H-Benzopyrans via Domino Reaction, Journal of Drug Delivery and Therapeutics. 2019; 9(4-A):271-279 http://dx.doi.org/10.22270/jddt.v9i4-A.3417

*Address for Correspondence:

S. Silambarasan, Department of Chemistry, Jamal Mohamed College, Tiruchirappalli 620020, Tamil Nadu, India.

\section{INTRODUCTION}

Chromene (benzopyran) is a heterocyclic ring system in which a benzene ring and a pyran ring are fused together. The chromene nucleus containing natural alkaloids are anthocyanins, tocopherols and flavonoids. Moreover, a variety of natural and synthetic chromene derivatives has an important biological and pharmacological applications, such as antimicrobial, [1] anti-inflammatory, [2] antiproliferative, [3] antioxidant, [4] herbicidal, analgesic and anticonvulsant, [5] antitubercular, [6] anticoagulant, estrogenic antispasmolytic, estrogenic, [7] TNF- $\alpha$ inhibitor effects and activities, [8] as well as inhibitor of diabetes-induced vascular dysfunction.[9] Moreover 7-Hydroxy-6-methoxy-
4H-chromene A (Fig. 1) is an example of naturally occurring $4 \mathrm{H}$-chromene, which was obtained from the flowers of Wisteria sinensis and is one of their fragrance components.[10] Among different types of chromene systems, 2-amino- $4 \mathrm{H}$-chromenes have been reported to exhibit highly useful proapoptotic properties for the treatment of a wide range of cancer ailments.[11] In cancer chemotherapy, 2-amino-4H-chromene $\mathbf{B}$ was marked for drug development due to its high inhibition of tumorassociated Bcl-2 proteins.[12] A modified 4H-chromene structure $\mathbf{C}$ was able to induce apoptosis (programmed cell death) in several cancer cell lines.[13] 
<smiles>N#CC1=C(N)OC=CC1</smiles><smiles>COc1cc2c(cc1O)OC(N)=C(C#N)C2</smiles>

Natural occurring 4h-chromen

(A)<smiles>COc1cc(C2C(C#N)=C(N)Oc3c2ccc2c3ccn2C)cc(Br)c1OC</smiles>

Induce apoptosis

(C)<smiles>N#CC1=C(N)Oc2c(c(=O)oc3ccccc23)C1c1ccccc1</smiles>

Antirheumatic<smiles>[R]c1cccc(C2C(C#N)=C(N)Oc3[nH]nc(C)c32)c1</smiles>

CH1-Kinase inhibitor<smiles>[R]c1ccc(C2C(C#N)=C(N)Oc3c2c(=O)oc2ccccc32)cc1</smiles>

$\mathrm{R}=3-\mathrm{NO}_{2} / 3.4-(\mathrm{OH})_{2}$ Anticancer<smiles>[R]c1cccc([C@H]2C(C#N)=C(N)Oc3c2c(C)nn3-c2ccc(F)cc2)c1</smiles><smiles>[R]c1cccc(C2C(C#N)=C(N)Oc3cc(N(C)C)ccc32)c1</smiles>

Anticancer<smiles>CCOCC(C#N)C1C(C(=O)OCC)=C(N)Oc2ccc(Br)cc21</smiles>

High inhibition of tumor-associated $\mathrm{Bcl}-2$ proteins

(B)<smiles></smiles>

$\mathrm{X}=\mathrm{O}$ / S: Anticancer $\mathrm{X}=\mathrm{NH}$ : Antibacterial<smiles>[R]c1cccc(C2C(C#N)=C(N)OC3=C2C(=O)CC(C)(C)C3)c1</smiles>

$\mathrm{R}=3-\mathrm{NO}_{2} / 3.4-(\mathrm{OH})_{2}$ Antibacterial

Figure 1: Structure of some biologically important 2-amino-3-cyano- $4 H$-chromenes

The biological records of these privileged motifs prompted us to develop an atom-economical, one pot, mild and greener method for the synthesis of chromene derivatives. Many synthetic procedures have appeared in the literature for the synthesis of 2-amino-7-hydroxy-4-phenyl-4H-chromene-3carbonitrile; these generally utilize the homogeneous, heterogeneous catalysts and acid/base mediated harsh reaction conditions. Therefore, a new synthetic route associated with environmentally friendly reaction condition is highly desirable. One of the most important progresses to combine economic aspects with the environmental concerns is the use of green protocol techniques which has provided simple and efficient synthetic methods of great promise. The elimination of toxic organic solvent in the reaction medium, shorter reaction time, straightforward work-up, and providing good to excellent yields with stoichiometric amounts of reactants are apparent advantages that distinguish the green chemical methods from others techniques such as microwave or ultrasound irradiation.[14,15] In the present work, we describe the synthesis of 2-amino-7-hydroxy-4-phenyl-4H-chromene-3carbonitrile derivatives via multi-component domino reaction in aqueous medium using aromatic aldehyde, resorcinol and malononitrile.

\section{RESULTS AND DISCUSSION}

Initially, multicomponent domino reaction of malononitrile, aryl aldehyde and resorcinol was chosen as the model reaction. The effect of various reaction parameters such as the influence of solvent, formation of hydrogen bond and the effect of temperature were studied to optimize the reaction conditions (Table 1). It is important to note that $\mathrm{K}_{2} \mathrm{CO}_{3}$ and $\mathrm{Na}_{2} \mathrm{CO}_{3}$ with a molar ratio of 0.1 was found to be the catalyst for the three component reaction of malanonitrile, aryl aldehyde and resorcinol affording a considerable yield of the desired product. Moreover in the absence of catalyst a significant product formation was observed under reflux condition (Table 1, entry 14).

The solvent temperature plays an important role in the reactivity (Table 1). We have investigated the effect of various protic, aprotic and non-polar solvents on the three component reaction of resorcinol, aryl aldehyde and malononitrile (Table 1, entry 1-14). In non-polar solvents such as 1,4-dioxane and Toluene, the yield of the reaction was found to be very low $(10-20 \%)$ (Table 1, entry $5,6,11,12)$. Whereas in the case of polar aprotic solvents such as THF, acetonitrile, the yield of the reaction was found to be low (8-15\%) (Table 1, entry $3,4,9,10)$. In the case of 
polar protic solvents such as ethanol and methanol (Table 1, entry 1,2,7,8), the yield of the desired product was considerable (50-65\%). Further increase in the reaction temperature to reflux makes the reaction almost quantitative (Table 1, entry 14). From Table 1, it is clear that water was the best choice as solvent.
We examined the substrate scope of substituted benzaldehydes (1a-1) under optimized reaction condition for the synthesis of chromene derivatives (4a-4l). All the electron donating and electron withdrawing aldehydes are compatible with optimized reaction condition afforded the excellent yield $80-93 \%$.

Table 1 Optimization of reaction conditions

\begin{tabular}{|c|c|c|c|c|c|}
\hline Entry & base & $\begin{array}{c}\text { solvent } \\
\text { (1 equiv) }\end{array}$ & Temp $\left({ }^{\circ} \mathrm{C}\right)$ & $\begin{array}{l}\text { Time } \\
\text { (min) }\end{array}$ & $\begin{array}{l}\text { Yield 4a } \\
(\%)\end{array}$ \\
\hline 1 & $\mathrm{Na}_{2} \mathrm{CO}_{3}$ & EtOH & 60 & 30 & 50 \\
\hline 2 & " & $\mathrm{MeOH}$ & 50 & 30 & 55 \\
\hline 3 & " & THF & 50 & 30 & 10 \\
\hline 4 & " & Acetonitrile & 60 & 30 & 8 \\
\hline 5 & " & Dioxane & 60 & 30 & 15 \\
\hline 6 & " & Toluene & 80 & 30 & 10 \\
\hline 7 & $\mathrm{~K}_{2} \mathrm{CO}_{3}$ & EtOH & 60 & 30 & 60 \\
\hline 8 & " & $\mathrm{MeOH}$ & 50 & 30 & 65 \\
\hline 9 & " & THF & 50 & 30 & 15 \\
\hline 10 & " & Acetonitrile & 60 & 30 & 10 \\
\hline 11 & " & Dioxane & 60 & 30 & 20 \\
\hline 12 & $"$ & Toluene & 80 & 30 & 12 \\
\hline 13 & - & $\mathrm{H}_{2} \mathrm{O}$ & 60 & 30 & 50 \\
\hline 14 & - & $\mathrm{H}_{2} \mathbf{O}$ & reflux & $15-20$ & $80-93$ \\
\hline
\end{tabular}

Scheme 1 Synthesise of 2-amino-3-cyano- $4 H$-chromenes derivatives (4a-l)<smiles>[R]c1cccc(C=O)c1</smiles>

1<smiles>N#CC1=C(N)Oc2cc(O)ccc2C1c1ccccc1</smiles>

4a, $90 \%, 15 \mathrm{~min}$<smiles>N#CC1=C(N)Oc2cc(O)ccc2C1c1cccc([N+](=O)[O-])c1</smiles>

4e, $93 \%, 20 \mathrm{~min}$<smiles>COc1cc(C2C(C#N)=C(N)Oc3cc(O)ccc32)cc(OC)c1OC</smiles>

4i, $93 \%, 10$ min<smiles>Oc1ccc(O)c(O)c1</smiles>

2<smiles>Cc1ccc(C2C(C#N)=C(N)Oc3cc(O)ccc32)cc1</smiles>

4b, $93 \%, 13 \mathrm{~min}$<smiles>COc1ccc(OC)c(C2C(C#N)=C(N)Oc3cc(O)ccc32)c1</smiles>

4f, $92 \%, 10 \mathrm{~min}$<smiles>N#CC1=C(N)Oc2cc(O)ccc2C1c1ccc(Cl)cc1</smiles>

4j, 90\%, 15 min<smiles>[R]c1cccc(C2C(C#N)=C(N)Oc3cc(O)ccc32)c1</smiles><smiles>N#CC1=C(N)Oc2cc(O)ccc2C1c1cccs1</smiles>

4c, $91 \%, 10 \mathrm{~min}$<smiles>COc1ccc(C2C(C#N)=C(N)Oc3cc(O)ccc32)cc1</smiles>

4d, $93 \%, 14 \mathrm{~min}$<smiles>COc1ccc(C2C(C#N)=C(N)Oc3cc(O)ccc32)cc1OC</smiles>

4g, $93 \%, 10 \mathrm{~min}$<smiles>N#CC1=C(N)Oc2cc(O)ccc2C1c1ccccc1Cl</smiles>

4k, $91 \%, 13$ min<smiles>N#CC1=C(N)Oc2cc(O)ccc2C1c1ccc(O)cc1</smiles>

4h, $92 \%, 13 \mathrm{~min}$<smiles>N#CC1=C(N)Oc2cc(O)ccc2C1c1cccc(Cl)c1Cl</smiles>

4I, $92 \%, 14 \mathrm{~min}$ 


\section{Single-crystal X-Ray study}

Refinement: Crystal data collection and structure refinement details are summarized in Table 2

Table 2. Crystallographic data for $\mathbf{4 d}$ and $\mathbf{4 k}$

\begin{tabular}{|c|c|c|}
\hline Compound & $4 \mathrm{~d}$ & $4 \mathrm{k}$ \\
\hline Identification code & mrb-4 & mrb-12 \\
\hline Empirical formula & $\mathrm{C}_{17} \mathrm{H}_{11} \mathrm{~N}_{2} \mathrm{O}_{3}$ & $\mathrm{C}_{16} \mathrm{H}_{11} \mathrm{C}_{1} \mathrm{~N}_{2} \mathrm{O}_{2}$ \\
\hline Formula weight & 287.30 & 270.31 \\
\hline Temperature & $293(2) \mathrm{K}$ & $296(2) \mathrm{K}$ \\
\hline Wavelength & $0.71073 \AA$ & $0.71073 \AA$ \\
\hline Crystal system & Triclinic, & Monoclinic, \\
\hline Space group & P1 & $\mathrm{P} 21 / \mathrm{n}$ \\
\hline Unit cell dimensions & $\begin{array}{ll}a=6.2316(3) \AA & \alpha=116.255(2)^{\circ} . \\
b=11.0207(6) \AA & \beta=90.099(2)^{\circ} \\
c=11.8732(6) \AA & \gamma=96.165(2)^{\circ} .\end{array}$ & $\begin{array}{l}\mathrm{a}=6.6658(3) \AA \quad \alpha=90^{\circ} \\
\mathrm{b}=30.1600(16) \AA \quad \beta=106.088(2)^{\circ} \\
\mathrm{c}=7.2193(4) \AA \quad \gamma=90^{\circ}\end{array}$ \\
\hline Volume & $725.95(6) \AA^{3}$ & $1394.53(12) \AA 3$ \\
\hline Z, Density (calculated) & $2,1.314 \mathrm{Mg} / \mathrm{m}^{3}$ & $4,1.287 \mathrm{Mg} / \mathrm{m} 3$ \\
\hline Absorption coefficient & $0.086 \mathrm{~mm}^{-1}$ & $0.078 \mathrm{~mm}^{-1}$ \\
\hline $\mathrm{F}(000)$ & 298 & 568 \\
\hline Crystal size & $0.20 \times 0.22 \times 0.20 \mathrm{~mm}$ & $0.15 \times 0.10 \times 0.10 \mathrm{~mm}$ \\
\hline Theta range for data collection & 3.72 to $36.41^{\circ}$ & 1.35 to $26.70^{\circ}$ \\
\hline Index ranges & $-10<=\mathrm{h}<=10,-18<=\mathrm{k}<=18,-19<=\mathrm{l}<=19$ & $-6<=\mathrm{h}<=8,-38<=\mathrm{k}<=38,-9<=\mathrm{l}<=8$ \\
\hline Reflections collected & $31554 / 6513[\mathrm{R}(\mathrm{int})=0.0402]$ & $21899 / 2942[\mathrm{R}(\mathrm{int})=0.0335]$ \\
\hline Completeness to theta $=$ & & $99.70 \%, 25.000^{\circ}$ \\
\hline Refinement method & $91.8 \%, 24.998^{\circ}$ & Full-matrix least-squares on $\mathrm{F}^{2}$ \\
\hline Data / restraints / parameters & Full-matrix least-squares on $\mathrm{F}^{2}$ & $2942 / 0 / 191$ \\
\hline Goodness-of-fit on $\mathrm{F}^{2}$ & & 1.111 \\
\hline Final $R$ indices $[\mathrm{I}>2 \operatorname{sigma}(\mathrm{I})]$ & 6513 / 2 / 209 & $\mathrm{R} 1=0.0407, \mathrm{wR} 2=0.1070$ \\
\hline R indices (all data) & 1.072 & $\mathrm{R} 1=0.0684, \mathrm{wR} 2=0.1384$ \\
\hline \multirow{4}{*}{ Largest diff. peak and hole } & & 0.319 and -0.210 e. $\AA^{-3}$ \\
\hline & $\mathrm{R} 1=0.1496, \mathrm{wR} 2=0.3302$ & \\
\hline & $\mathrm{R} 1=0.1990, \mathrm{wR} 2=0.3606$ & \\
\hline & 1.069 and -0.760 e. $\AA^{-3}$ & \\
\hline
\end{tabular}

The X-ray diffraction analysis revels that the benzopyran ring of the compound $\mathbf{4 d}, \mathrm{C}_{17} \mathrm{H}_{11} \mathrm{~N}_{2} \mathrm{O}_{3}$, is planar [maximum deviation $=0.080$ (2) $\AA$ ] and the methoxyphenyl ring is also in planar conformation. The benzopyran derivative is almost perpendicular to the methoxyphenyl ring [dihedral angle = $\left.88.75(5)^{\circ}\right]$. The ORTEP plot of the molecule is shown in Fig.2. The methoxyphenyl substituted benzopyran compound crystallise in triclinic P1 space group. In the structure, the benzopyran and methoxyphenyl rings are planar. The benzopyran ring makes a dihedral angle of $90.857(5)^{\circ}$ with the methoxyphenyl ring and confirms the fact that the two moieties are in axial orientation. The orientation of the benzopyran and methoxyphenyl rings also confirmed from the torsion angle values of [C3-C4-C12$\mathrm{C} 13=] 75.6(2)^{\circ}$ \& [C3-C4-C12-C17=] -105.3 (2) ${ }^{\circ}$, respectively. In the benzopyran moiety, the attached carbonitrile, amino and hydroxy groups lie in a same plane, with the maximum deviation of atom N2[-0.055 (2) $\AA]$. The sum of the bond angles around atom $\mathrm{N} 1$ of the pyran ring is in accordance with $\mathrm{sp}^{2}$ hybridization state[360$]$

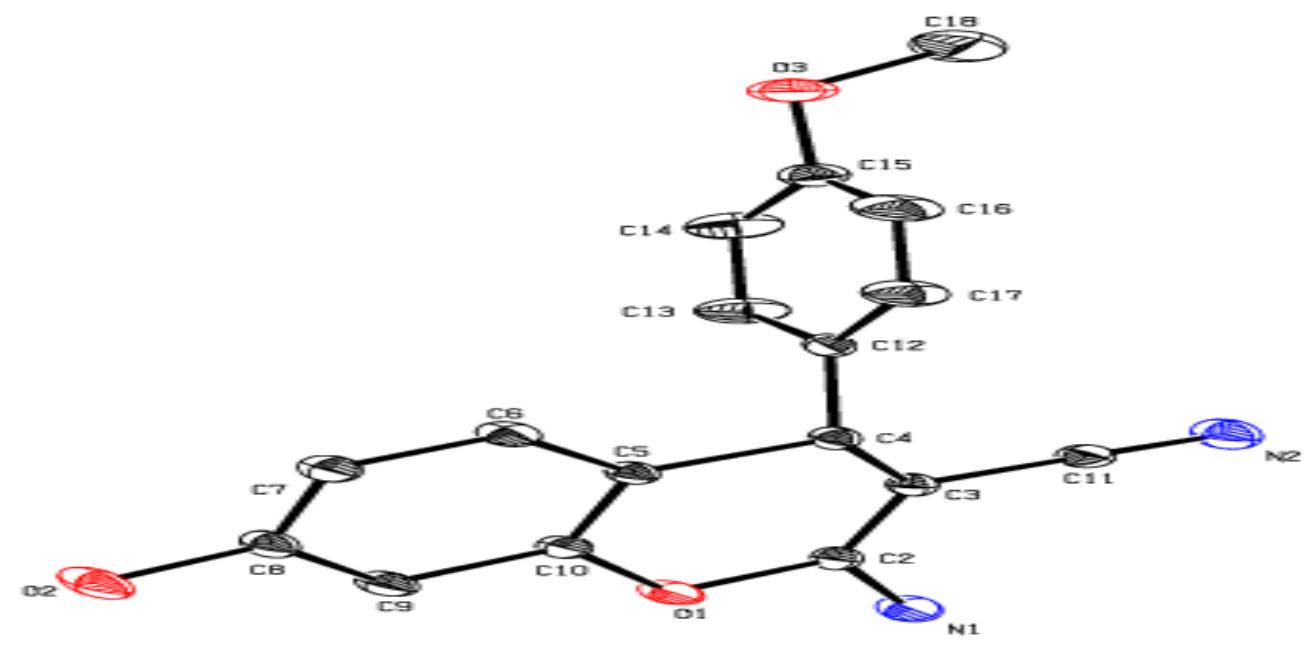

Figure 2: ORTEP diagram of $4 d$ 
The benzopyran ring of the compound $4 \mathbf{k}, \mathrm{C}_{16} \mathrm{H}_{11} \mathrm{~N}_{2} \mathrm{O}_{2} \mathrm{Cl}$, is planar [maximum deviation $=0.079$ (2) $\AA$ ] and the chlorophenyl ring is also in planar conformation. The benzopyran derivative is almost perpendicular to the chlorophenyl ring [dihedral angle $=86.85(6)^{\circ}$ ]. The ORTEP plot of the molecule is shown in Fig.3. The chlorophenyl substituted benzopyran compound crystallise in monoclinic P2 1/c space group. In the structure, the benzopyran and chlorophenyl rings are planar. The benzopyran ring makes a dihedral angle of $86.85(6)^{\circ}$ with the chlorophenyl ring and confirms the fact that the two moieties are in axial orientation. The chlorophenyl moiety is also planar with the maximum deviation noted for atom CL12 as -0.040 (1) Å. The orientation of the benzopyran and chlorophenyl rings also confirmed from the torsion angle values of [C3-C4C11-C12=] $76.5(2)^{\circ} \&$ [C3-C4-C11-C16=] -100.4 (2) , respectively. In the benzopyran moiety, the attached carbonitrile, amino and hydroxy groups lie in a same plane, with the maximum deviation of atom N2[-0.053 (2) $\AA]$. The sum of the bond angles around atom N1 of the pyran ring is in accordance with $\mathrm{sp}^{2}$ hybridization state [360].

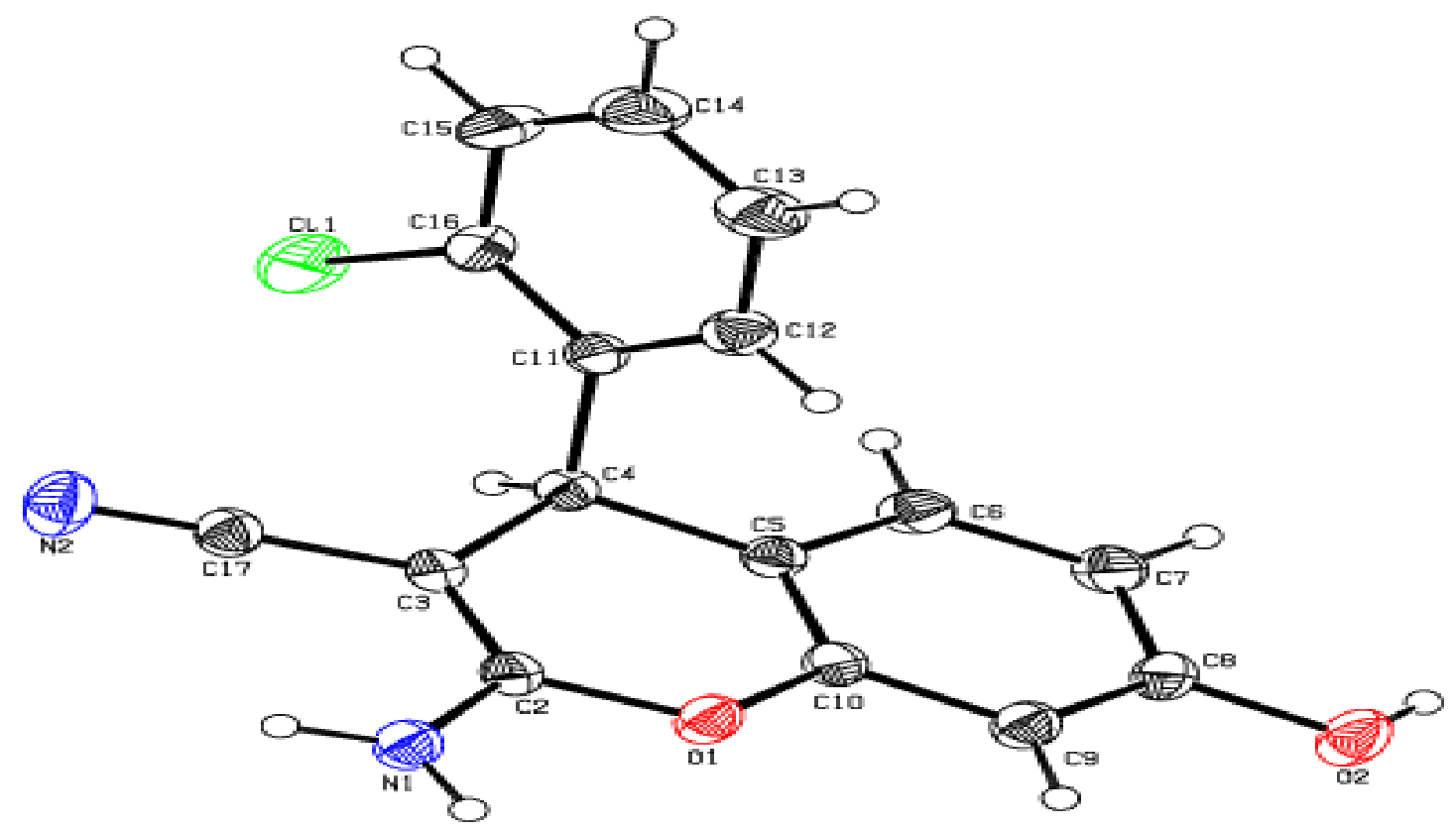

Figure 3: ORTEP diagram of $4 k$

\section{Experimental}

All the chemicals used were purchased from Merck AnalaR grade and purified wherever necessary using the standard purification method. The purity of compounds were checked by TLC using silica gel-G plate and visualized by iodine vapours. The melting points were recorded in open capillary tube and uncorrected. The FT-IR spectra were recorded in SHIMADZU FT-IR Affinity-I spectrometer using $\mathrm{KBr}$ pellets and Perkinealmer. The ${ }^{1} \mathrm{H}-\mathrm{NMR}$ and ${ }^{13} \mathrm{C}$-NMR spectra were obtained in DMSO- $\mathrm{d}_{6}$ on BRUKER $300 \mathrm{MHZ}$ and 500MHZ instrument with TMS as an internal standard and the chemical shift values are presented in ppm. The mass spectra were taken on SHIMADZU GC-MS QP 2010 spectrometer operating at an ionization potential of $70 \mathrm{ev}$.

General experimental procedure for the Synthesis of 2amino-7-hydroxy-4-phenyl-4H-chromene-3-carbonitrile

To a stirred solution of substituted benzaldehyde ( $0.05 \mathrm{~mol})$ in water was added resorcinol $(0.05 \mathrm{~mol})$ and malononitrile $(0.05 \mathrm{~mol})$ at room temperature and reflexed for $15-20$ minutes. The progress of the reaction was monitored by thin layer chromatography using silica gel-G plates. The reaction mixture was cooled and allowed to 30 minutes. The precipitated solid was filtered at suction pump. The crude solid obtained was recrystalized by methanol.

\section{2-Amino-7-hydroxy-4-phenyl-4H-chromene-3- carbonitrile $4 \mathrm{a}$}

IR: (KBr cm-1) $3425(\mathrm{OH}), 3332,3219\left(\mathrm{NH}_{2}\right), 2193(\mathrm{CN}), 1651$ (C=C Vinyl nitrile) 1587 (C=C Aromatic), $\mathrm{H}^{1} \mathrm{NMR}(300 \mathrm{MHz}$ DMSO - $\left.\mathrm{d}_{6}\right): \delta_{\mathrm{H}} 4.63(\mathrm{~S}, 1 \mathrm{H}), 6.43(\mathrm{~d}, J=2.4,1 \mathrm{H}), 6.48-6.52(\mathrm{~m}$, 1H) $6.81(\mathrm{~d}, J=8.7,1 \mathrm{H}), 6.89(\mathrm{~s}, 2 \mathrm{H}) 7.16-7.22(\mathrm{~m}, 3 \mathrm{H}), 7.29$ $(\mathrm{t}, J=6.9,2 \mathrm{H}), 9.74(\mathrm{~s}, 1 \mathrm{H}),{ }^{13} \mathrm{C}$ NMR $(300 \mathrm{MHz}$ DMSO-d $)$; $\delta_{\mathrm{c}}$ 56.22, 102.15, 112.36, 113.71, 120.68, 126.61, 127.35, $128.56,129.90,146.35,148.82,157.06,161.23$.

\section{2-Amino-7-hydroxy-4-(p-tolyl)-4H-chromene-3-} carbonitrile $4 \mathrm{~b}$

IR: ( $\left.\mathrm{KBr} \mathrm{cm}^{-1}\right) 3410(\mathrm{OH}), 3340,3230\left(\mathrm{NH}_{2}\right), 2181(\mathrm{CN}), 1641$ (C=C Vinyl nitrile) 1587 ( $\mathrm{C}=\mathrm{C}$ Aromatic), $\mathrm{H}^{1} \mathrm{NMR}(300 \mathrm{MHz}$ ): $\delta_{\mathrm{H}} 2.24(\mathrm{~s}, 3 \mathrm{H}), 4.58(\mathrm{~s}, 1 \mathrm{H}), 6.44(\mathrm{~d}, J=2.1,1 \mathrm{H}), 6.48-6.52(\mathrm{~m}$, $1 \mathrm{H}), 6.78(\mathrm{~d}, J=8.4,1 \mathrm{H}), 6.86(\mathrm{~s}, 2 \mathrm{H}), 7.04-7.12(\mathrm{~m}, 4 \mathrm{H}), 9.72$ (s, 1H), ${ }^{13}$ C NMR (300 MHz DMSO-d6); $\delta_{c} 20.55,56.39,102.12$, $112.31,113.87,120.70, \quad 127.28, \quad 129.10, \quad 135.68$, $143.40,148.78,156.99,160.14$.

2-Amino-7-hydroxy-4-(thiophen-2-yl)-4H-chromene-3carbonitrile 4c

IR: ( $\left.\mathrm{KBr} \mathrm{cm}^{-1}\right)$ 3421(OH), 3332, 3219 ( $\left.\mathrm{NH}_{2}\right), 2193(\mathrm{CN}), 1654$ ( $\mathrm{C}=\mathrm{C}$ Vinyl nitrile) 1589 ( $\mathrm{C}=\mathrm{C}$ Aromatic), $\mathrm{H}^{1} \mathrm{NMR}(300 \mathrm{MHz}$ DMSO -d6): $\delta_{\mathrm{H}} 4.9(\mathrm{~s}, 1 \mathrm{H}), 6.40(\mathrm{~d}, J=2.4,1 \mathrm{H}), 6.52-6.56(\mathrm{~m}$, $1 \mathrm{H}), 6.91-6.94(\mathrm{~m}, 1 \mathrm{H}), 6.97(\mathrm{t}, J=3.6,2 \mathrm{H}), 6.99(\mathrm{~s}, 2 \mathrm{H}), 7.34$ 
7.36 (m, 1H), 9.77 (s, 1H), ${ }^{13} \mathrm{C}$ NMR (300 MHz DMSO-d6); $\delta_{c}$ $56.39,102.15,112.37,113.49,120.50,124.00,125.02$, $126.72,129.85,148.51,151.48,157.30,160.28$.

\section{2-Amino-7-hydroxy-4-(4-methoxyphenyl)-4H-} chromene-3-carbonitrile $4 \mathrm{~d}$

IR: (KBr cm-1) 3415(OH), 3334, $3221\left(\mathrm{NH}_{2}\right), 2187(\mathrm{CN}), 1655$ (C=C Vinyl nitrile) 1587 (C=C Aromatic), $\mathrm{H}^{1} \mathrm{NMR}(300 \mathrm{MHz}$ DMSO - $\left.\mathrm{d}_{6}\right): \delta_{\mathrm{H}} 3.71(\mathrm{~s}, 3 \mathrm{H}), 4.58(\mathrm{~s}, 1 \mathrm{H}), 6.42(\mathrm{~d}, J=2.1,1 \mathrm{H})$, 6.48-6.52 (m, 1H), 6.85-7.13 (m, 6H), $9.71(\mathrm{~s}, 1 \mathrm{H}),{ }^{13} \mathrm{C}$ NMR (300 MHz DMSO-d 6 ); $\delta_{\mathrm{c}} 54.96,56.56,102.09,112.30,113.88$, $114.03,120.72,128.41,129.91,130.13,138.46,148.73$ $156.94,157.93,160.05$

\section{2-Amino-7-hydroxy-4-(3-nitrophenyl)-4H-chromene-3- carbonitrile $4 \mathrm{e}$}

IR: (KBr cm-1) 3450(OH), 3334, $3221\left(\mathrm{NH}_{2}\right), 2190(\mathrm{CN}), 1658$ (C=C Vinyl nitrile) 1587 (C=C Aromatic), $\mathrm{H}^{1} \mathrm{NMR}(300 \mathrm{MHz}$ DMSO - $\left.\mathrm{d}_{6}\right): \delta_{\mathrm{H}} 4.96(\mathrm{~s}, 1 \mathrm{H}), 6.52(\mathrm{~d}, J=2.4 \mathrm{~Hz}, 1 \mathrm{H}), 5.5-5.58$ $(\mathrm{m}, 1 \mathrm{H}), 6.87(\mathrm{~d}, J=8.4 \mathrm{~Hz}, 1 \mathrm{H}), 7.11(\mathrm{~s}, 2 \mathrm{H}), 7.63-7.72(\mathrm{~m}$, $2 \mathrm{H}), 8.08-8.14(\mathrm{~m}, 2 \mathrm{H}), 9.87(\mathrm{~s}, 1 \mathrm{H}) ;{ }^{13} \mathrm{C}$ NMR $(300 \mathrm{MHz}$ DMSO-d6); $\delta_{\mathrm{c}} 55.31,112.49,112.66,120.40,121.74,121.82$, $129.96,130.27,134.27,147.89,148.53,148.88$.

\section{2-Amino-4-(2,5-dimethoxyphenyl)-7-hydroxy-4H-} chromene-3-carbonitrile $4 \mathrm{f}$

IR: ( $\left.\mathrm{KBr} \mathrm{cm}^{-1}\right)$ 3406(OH), 3338, $3223\left(\mathrm{NH}_{2}\right), 2187(\mathrm{CN}), 1657$ (C=C Vinyl nitrile) 1503 (C=C Aromatic), $\mathrm{H}^{1} \mathrm{NMR}(300 \mathrm{MHz}$ DMSO - $\left.\mathrm{d}_{6}\right): \delta_{\mathrm{H}} 3.63(\mathrm{~s}, 3 \mathrm{H}), 3.71(\mathrm{~s}, 3 \mathrm{H}), 4.93(\mathrm{~s}, 3 \mathrm{H}), 6.39(\mathrm{~d}$, $J=2.0 \mathrm{~Hz}, 1 \mathrm{H}), 6.46-6.48(\mathrm{~m}, 1 \mathrm{H}), 6.53(\mathrm{~d}, J=3.0 \mathrm{~Hz}, 1 \mathrm{H}), 6.73-$ $6.76(\mathrm{~m}, 1 \mathrm{H}), 6.82(\mathrm{~s}, 2 \mathrm{H}), 6.84(\mathrm{~d}, J=8.5 \mathrm{~Hz}, 1 \mathrm{H}), 6.90(\mathrm{~d}, J=9.0$ $\mathrm{Hz}, 1 \mathrm{H}) 9.67(\mathrm{~s}, 1 \mathrm{H}),{ }^{13} \mathrm{C}$ NMR $(300 \mathrm{MHz}$ DMSO-d 6$) ; \delta_{\mathrm{c}} 55.56$, 55.66, 56.71, 102.56, 111.98, 112.65, 113.26, 114.25, 115.54, $121.24,129.69,135.78,149.57,151.06,153.75,157.39$, 161.41 .

\section{2-Amino-4-(3,4-dimethoxyphenyl)-7-hydroxy-4H- chromene-3-carbonitrile $4 \mathrm{~g}$}

IR: (KBr cm$\left.{ }^{-1}\right)$ 3435(OH), 3332, $3233\left(\mathrm{NH}_{2}\right), 3070(\mathrm{CH}-\mathrm{Ar})$, 2135(CH-Aliphatic), 2194 (CN), 1643 (C=C Vinyl nitrile) 1571 (C=C Aromatic), $\mathrm{H}^{1}$ NMR (300 MHz DMSO - d6): $\delta_{\mathrm{H}} 3.84$ (s, 3H), $3.98(\mathrm{~s}, 3 \mathrm{H}), 4.59(\mathrm{~s}, 1 \mathrm{H}), 6.42(\mathrm{~d}, J=2.4 \mathrm{~Hz}, 1 \mathrm{H}), 6.50-$ $6.52(\mathrm{~m}, 1 \mathrm{H}), 6.67-6.70(\mathrm{~m}, 1 \mathrm{H}), 6.80(\mathrm{~d}, J=1.8 \mathrm{~Hz}, 1 \mathrm{H}), 6.83$ (s, 2H), 6.86-6.90(m, 2H), $9.71(\mathrm{~s}, 1 \mathrm{H}),{ }^{13} \mathrm{C}$ NMR $(300 \mathrm{MHz}$ DMSO-d $\left.{ }_{6}\right) ; \delta_{\mathrm{c}} 54.66,55.40,56.38,102.08,111.18,112.27$, $113.91,115.80,121.46,125.97,129.88,138.84,147.53$, $148.23,150.30,156.93,160.14,161.24,165.85$.

2-Amino-7-hydroxy-4-(4-hydroxyphenyl)-4H-chromene3-carbonitrile $4 \mathrm{~h}$

IR: ( $\left.\mathrm{KBr} \mathrm{cm}^{-1}\right)$ 3481(OH), 3354, $3254\left(\mathrm{NH}_{2}\right), 2193(\mathrm{CN}), 1652$ (C=C Vinyl nitrile) 1588 (C=C Aromatic), $\mathrm{H}^{1} \mathrm{NMR}(300 \mathrm{MHz}$ DMSO $\left.-\mathrm{d}_{6}\right): \delta_{\mathrm{H}} 4.49(\mathrm{~s}, 1 \mathrm{H}), 3.98(\mathrm{~s}, 3 \mathrm{H}), 4.49(\mathrm{~s}, 1 \mathrm{H}), 6.39(\mathrm{~s}$, $1 \mathrm{H}), 6.47(\mathrm{~d}, J=8.5 \mathrm{~Hz}, 1 \mathrm{H}), 6.67(\mathrm{~d}, J=8 \mathrm{~Hz}, 2 \mathrm{H}), 6.67(\mathrm{~s}, 1 \mathrm{H})$, $6.78(\mathrm{~s}, 2 \mathrm{H}), 6.95(\mathrm{~d}, J=8.5 \mathrm{~Hz}, 2 \mathrm{H}),, 9.28(\mathrm{~s}, 1 \mathrm{H}),{ }^{13} \mathrm{C} \mathrm{NMR}$ (300 MHz DMSO-d $)$; $\delta_{c} 57.23,102.53,112.73,114.78$, $115.68,121.24,128.83,130.36,137.27,149.22,156.50$ $157.33,160.51$.

\section{2-Amino-7-hydroxy-4-(3,4,5-trimethoxyphenyl)-4H- chromene-3-carbonitrile 4i}

IR: (KBr cm-1) 3410(OH), 3333, $3222\left(\mathrm{NH}_{2}\right), 3073(\mathrm{CH}-\mathrm{Ar})$, 2945(CH-Aliphatic), 2194 (CN), 1658 (C=C Vinyl nitrile) 1592 (C=C Aromatic), H ${ }^{1}$ NMR (300 MHz DMSO -d6): $\delta$ H 3.62 $(\mathrm{s}, 3 \mathrm{H}), 3.71(\mathrm{~s}, 6 \mathrm{H}), 4.58(\mathrm{~s}, 1 \mathrm{H}), 6.39(\mathrm{~s}, 1 \mathrm{H}), 6.47-6.50(\mathrm{~m}$, $3 \mathrm{H}), 6.85(\mathrm{~s}, 2 \mathrm{H}), 6.85(\mathrm{~d}, J=8.5 \mathrm{~Hz}, 1 \mathrm{H}), 9.69(\mathrm{~s}, 1 \mathrm{H}),{ }^{13} \mathrm{C}$ NMR (300 MHz DMSO-d 6 ); $\delta_{c} 56.25,56.37,60.37,102.60,105.01$, $112.79,113.97,121.18,130.33,136.61,142.44,149.12$, $157.51,160.80$.

\section{2-Amino-4-(2,3-dichlorophenyl)-7-hydroxy-4H- chromene-3-carbonitrile $4 \mathrm{j}$}

IR: (KBr cm-1) $3406(\mathrm{OH}), 3342,3266\left(\mathrm{NH}_{2}\right), 3035(\mathrm{CH}-\mathrm{Ar})$, 2928 (CH-Aliphatic), $2195 \quad(\mathrm{CN}), 1643 \quad(\mathrm{C}=\mathrm{C}) 1461$ (C=C),1154 (C-C), 1109 (C-O-C), 1342 (C-N), H ${ }^{1}$ NMR (500 MHz DMSO - d6): $\delta_{H} 5.21(\mathrm{~s}, 1 \mathrm{H}), 6.41(\mathrm{~d}, J=20.5 \mathrm{~Hz}, 1 \mathrm{H}), 6.42-$ $6.49(\mathrm{~m}, 1 \mathrm{H}), 6.72(\mathrm{~d}, J=8.5 \mathrm{~Hz}, 1 \mathrm{H}), 6.99(\mathrm{~s}, 2 \mathrm{H}), 7.17(\mathrm{~d}$, $J=7.5 \mathrm{~Hz}, 1 \mathrm{H}), 7.31-7.34(\mathrm{~m}, 1 \mathrm{H}), 7.51(\mathrm{~d}, J=8 \mathrm{~Hz}, 1 \mathrm{H}), 9.80(\mathrm{~s}$ $1 \mathrm{H}),{ }^{13} \mathrm{C}$ NMR $\left(500 \mathrm{MHz}\right.$ DMSO $\left.\mathrm{d}_{6}\right), \delta_{\mathrm{c}} 54,102.75,112.36$, $113.05,120.67,129.09,129.62,129.67,130.03,130.45$, $149.50,157.96,161.00$.

\section{2-Amino-4-(4-chlorophenyl)-7-hydroxy-4H-chromene- 3-carbonitrile 4k}

IR: (KBr cm$\left.{ }^{-1}\right) 3464(\mathrm{OH}), 3340,3243\left(\mathrm{NH}_{2}\right), 3063$ (CH-Ar), 2928 (CH-Aliphatic), 2192 (CN), 1639 (C=C) 1458 (C=C), 1155 (C-C), 1110 (C-O-C), 1344 (C-N), H1 NMR (500 MHz DMSO -d6): $\delta_{\mathrm{H}} 6.41(\mathrm{~d}, 1 \mathrm{H}, \mathrm{J}=2 \mathrm{~Hz}, \mathrm{H}-\mathrm{Ar}$ ), 6.48-6.50 (dd, $1 \mathrm{H}$ $J=2,6.5 \mathrm{~Hz}, \mathrm{H}-\mathrm{Ar}$ ), 6.77 (d, 1H, $J=8.5 \mathrm{~Hz}, \mathrm{H}-\mathrm{Ar}), 6.91$ (S, 2H, $\mathrm{NH}_{2}$ ), 7.18 (d, 2H, J=8.5 Hz, H-Ar), 7.35 (d, 2H, J=8.5 Hz, HAr), $9.72(\mathrm{~S}, 1 \mathrm{H}, \mathrm{OH}),{ }^{13} \mathrm{C}$ NMR ( $500 \mathrm{MHz}$ DMSO $\left.\mathrm{d}_{6}\right), \delta_{\mathrm{c}} 56.35$, $102.71,112.95,113.67,120.97,129.03,129.75,130.36$, $131.71,145.76,149.30,157.69,160.72$.

\section{2-Amino-4-(2-chlorophenyl)-7-hydroxy-4H-chromene-} 3-carbonitrile 41

IR: (KBr cm-1) $3421(\mathrm{OH}), 3337,3220\left(\mathrm{NH}_{2}\right), 3070$ (CH-Ar), 2922 (CH-Aliphatic), 2193 (CN), 1653 (C=C) 1461 (C=C), 1110 (C-O-C), 1301 (C-N), H ${ }^{1}$ NMR (500 MHz DMSO -d $)$ : $\delta_{H}$ 6.42 (d, $1 \mathrm{H}, J=1.5 \mathrm{~Hz}, \mathrm{H}-\mathrm{Ar}$ ), 6.47-6.49 (dd, $1 \mathrm{H}, J=2.5,6 \mathrm{~Hz}, \mathrm{H}-$ Ar), 6.73 (d, 1H, $J=8.5 \mathrm{~Hz}, \mathrm{H}-\mathrm{Ar}$ ), 6.92 (s, 2H, NH2), 7.179$7.19(\mathrm{t}, 1 \mathrm{H}, J=7 \mathrm{~Hz}, \mathrm{H}-\mathrm{Ar}), 7.21-7.24(\mathrm{q}, 1 \mathrm{H}, J=1.5,6 \mathrm{~Hz}, \mathrm{H}-$ Ar), 7.28-7.31 (t, 1H, J=7.5 Hz, Ar-CH), 7.40 (d, $1 \mathrm{H}, 7.45 \mathrm{~Hz}$, $\mathrm{Ar}-\mathrm{H}), 9.74(\mathrm{~S}, 1 \mathrm{H}, \mathrm{OH}),{ }^{13} \mathrm{C}$ NMR $\left(500 \mathrm{MHz}\right.$ DMSO $\left.\mathrm{d}_{6}\right), \delta_{\mathrm{c}}$ $55.37,102.72,112.96,112.97,120.76,128.28,129.00$, $129.71,130.18,131.24,132.30,143.31,149.48,157.80$, 160.98 . 


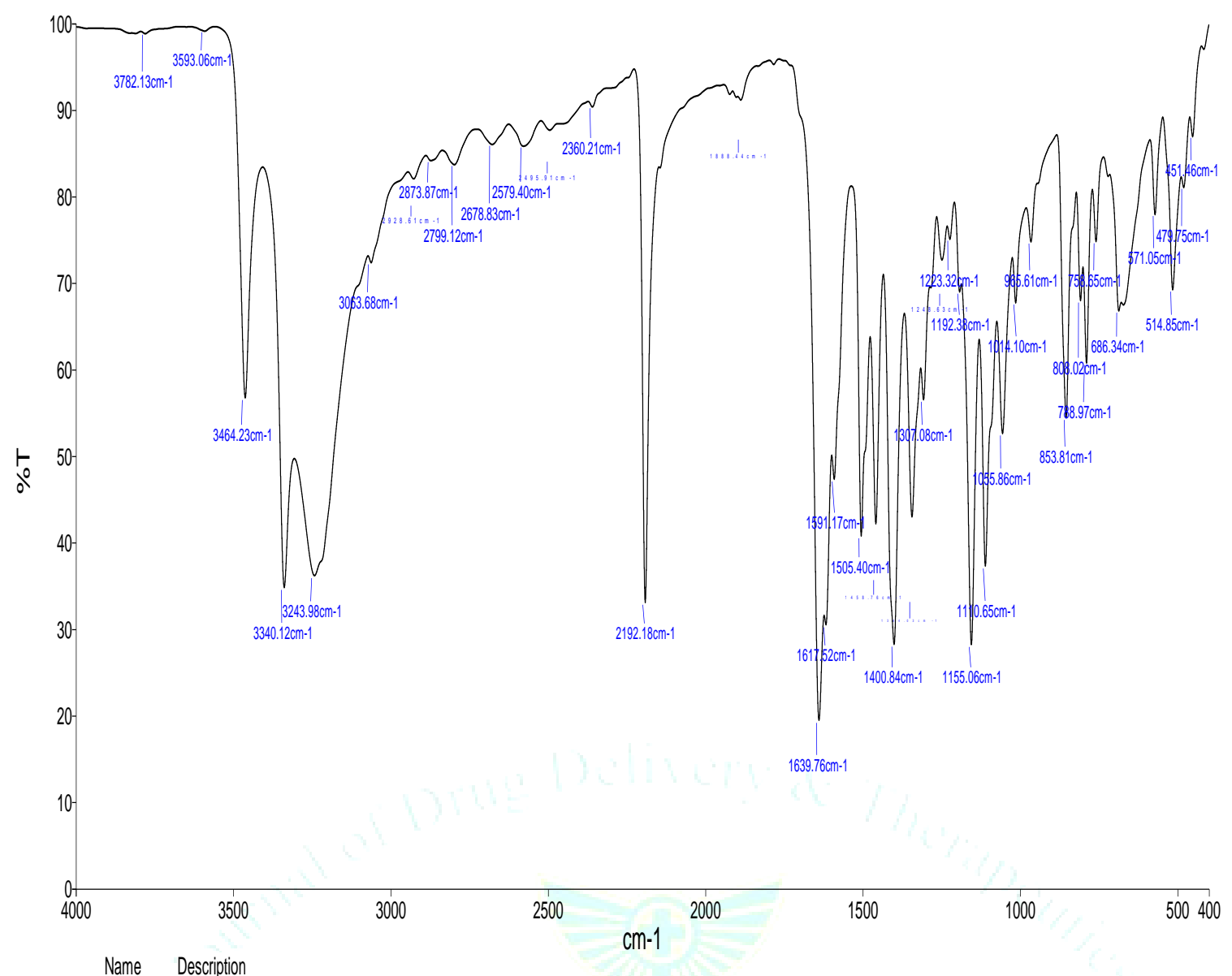

SS-MRB-11-

Figure 4: IR-Spectrum of 2-amino-4-(4-chlorophenyl)-7-hydroxy-4H-chromene-3-carbonitrile 4k

MRB-11
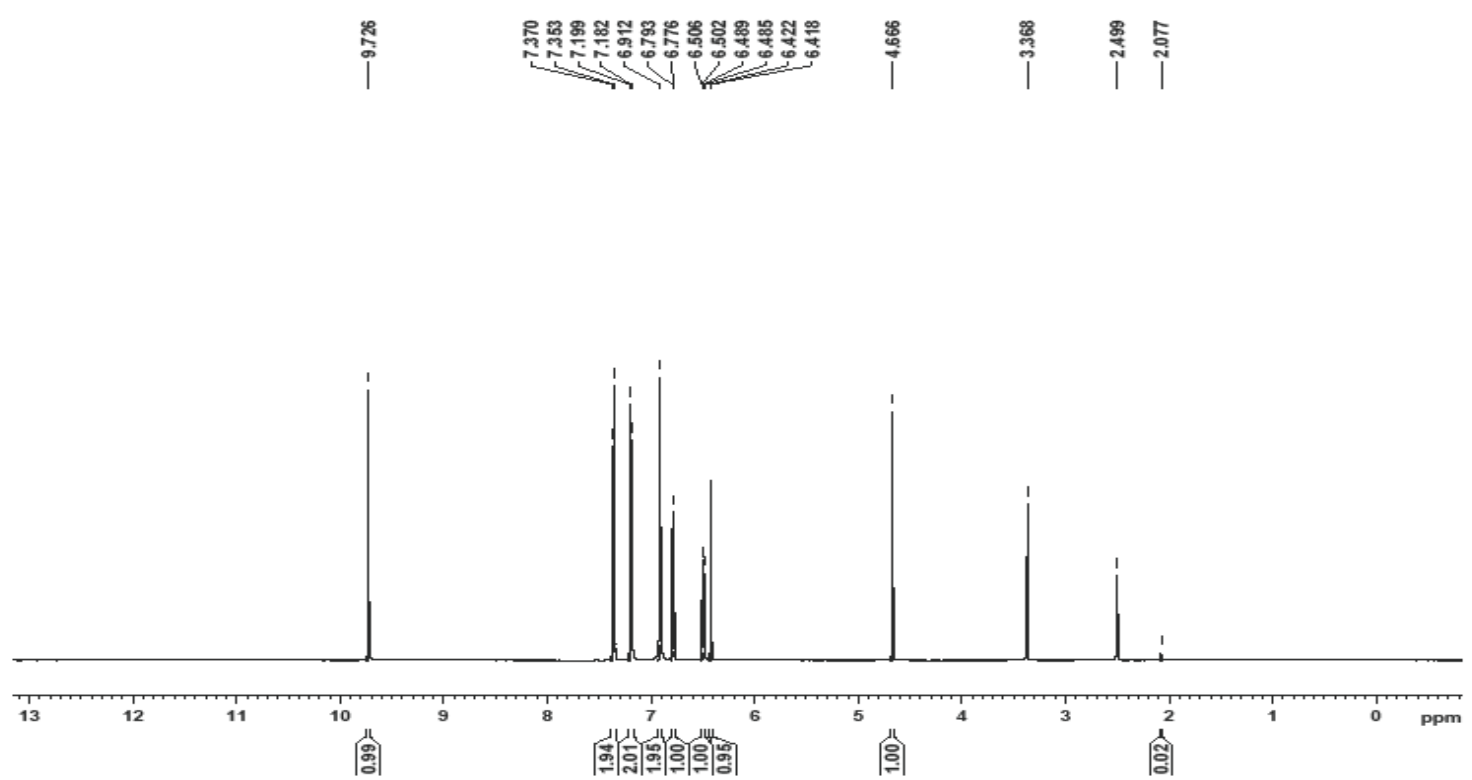

Figure 5: ${ }^{1} \mathrm{H}$ NMR-Spectrum of 2-amino-4-(4-chlorophenyl)-7-hydroxy-4H-chromene-3-carbonitrile 4k 
MRB-11
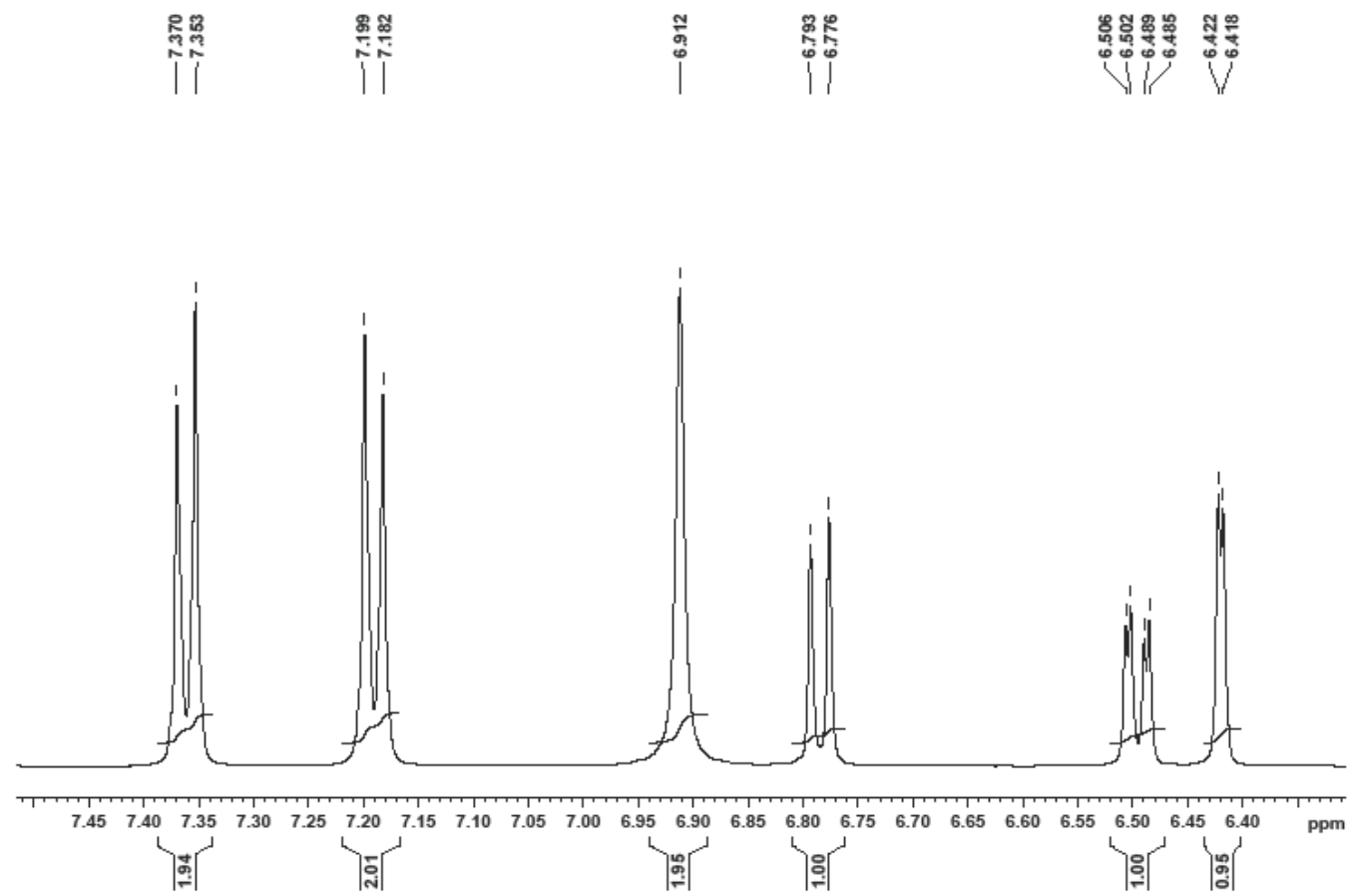

Figure 5a: 1H NMR-Spectrum of 2-amino-4-(4-chlorophenyl)-7-hydroxy-4H-chromene-3-carbonitrile 4k

MRB-11
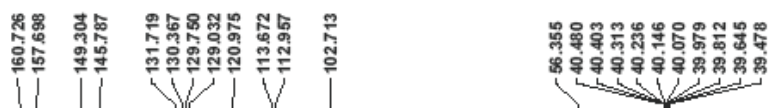

\$

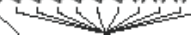

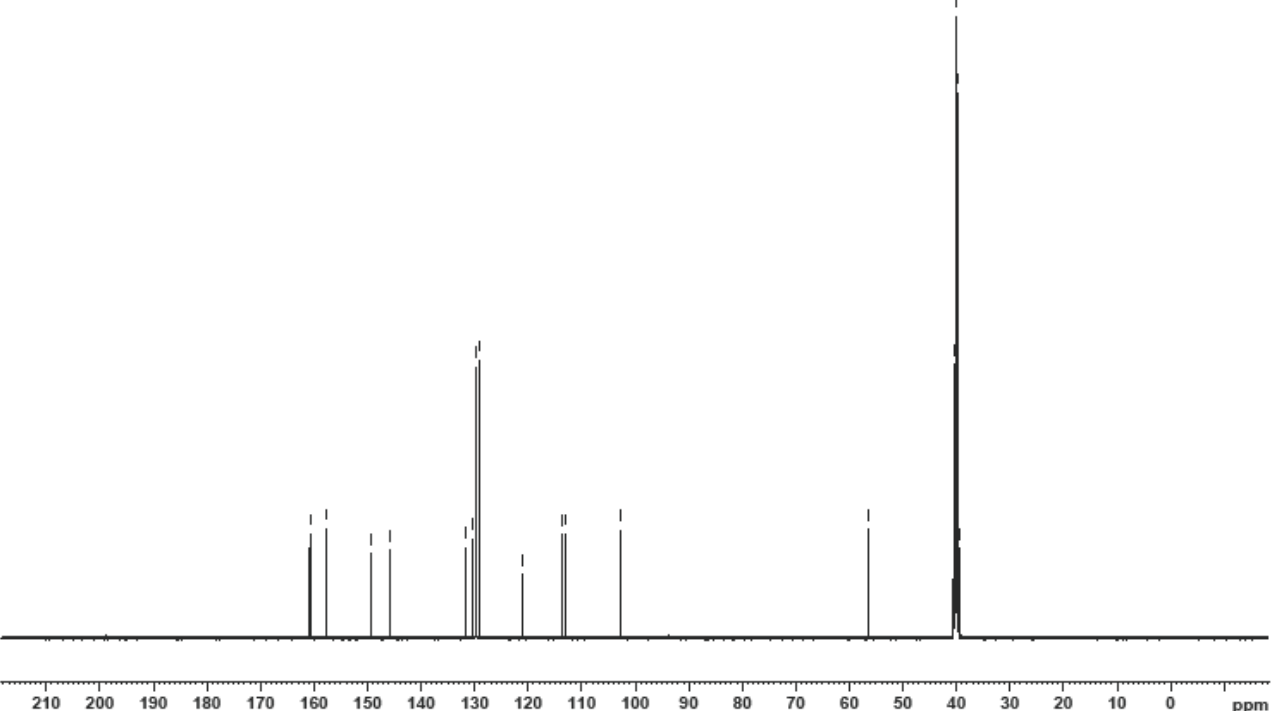

Figure 6: 13C NMR-Spectrum of 2-amino-4-(4-chlorophenyl)-7-hydroxy-4H-chromene-3-carbonitrile 4k

\section{CONCLUSION}

In this work, cascade reaction has been carried out for the synthesis of 2-amino-3-cyanobenzopyrans $4 \mathrm{k}$ from malononitrile, aryl aldehyde and resorcinol in methanol, ethanol, acetone and water solvents using $\mathrm{K}_{2} \mathrm{CO}_{3}$ or $\mathrm{Na}_{2} \mathrm{CO}_{3}$ as base. Nevertheless, the use of water was successful for this reaction. Under the found conditions, the high yield for the products were achieved at short reaction time in aqueous medium. The structure of the products were confirmed by IR, ${ }^{1} \mathrm{H},{ }^{13} \mathrm{C}$ NMR spectra and X-ray diffraction study. 


\section{REFERENCES}

[1] Kathrotiya HG, Patel MP, Microwave-assisted synthesis of 30indolyl substituted $4 \mathrm{H}$-chromenes catalyzed by DMAP and their antimicrobial activity, Medicinal Chemistry Research, 2012; 21:3406-3416.

[2] Thomas N, Zachariah SM, In Silico drug design and analysis of 4-Phenyl-4H-chromene derivatives as anticancer and antiinflammatory agents, International Journal of Pharmaceutical Sciences Review and Research, 2013; 22:50-54.

[3] Magedov IV, Manpadi M, Evdokimov NM, Elias EM, Rozhkova E, Ogasawara MA, Bettale JD, Przheval'skii NM, Rogelj S, Kornienko A, Antiproliferative and apoptosis inducing properties of pyrano[3,2-c]pyridones accessible by a one-step multicomponent synthesis, Bioorganic \& Medicinal Chemistry Letters, 2007; 17:3872-3876.

[4] Singh OM, Devi NS, Thokchom DS, Sharma GJ, Novel 3alkanoyl/aroyl/-heteroaroyl-2H-chromene-2-thiones: synthesis and evaluation of their antioxidant activities, European Journal of Medicinal Chemistry, 2010; 45:22502257.

[5] Bhat M, Siddiqui N, Khan S, Synthesis of novel 3-(4-acetyl5H/methyl-5-substituted phenyl-4,5-dihydro-1,3,4-oxadiazol2-yl)-2H-chromen-2-ones as potential anticonvulsant agents, Acta poloniae pharmaceutica, 2008; 65:235-239.

[6] Nimesh RK, Dhaval DH, Prashant TM, Saurabh KP, Synthesis and evaluation of in vitro antitubercular activity and antimicrobial activity of some novel 4H-chromeno[2,3 d]pyrimi-dine via 2-amino-4-phenyl-4H-chromene-3carbonitriles, Medicinal Chemistry Research, 2011; 20:854864 .

[7] Nareshkumar J, Jiayi X, Ramesh MK, Fuyong D, Guo JZ, Emmanuel $\mathrm{P}$, Identification and structure-activity relationships of chromene-derived selective estrogen receptor modulators for treatment of postmenopausal symptoms, Journal of Medicinal Chemistry, 2009; 52: 7544-7569.

[8] Cheng JF, Ishikawa A, Ono Y, Arrhenius T, Nadzan A, Novel chromene derivatives as TNF-a inhibitors, Bioorganic \& Medicinal Chemistry Letters, 2003; 13:3647-3650.

[9] Birch KA, Heath WF, Hermeling RN, Johnston CM, Stramm L, Dell C, Smith C, Williamson JR, Reifel-Miller A, LY290181, an inhibitor of diabetes induced vascular dysfunction, blocks protein kinase $\mathrm{C}$-stimulated transcriptional activation through inhibition of transcription factor binding to a phorbol response element, Diabetes, 1996; 45:642-650.

[10] Joulain D, Tabacchi R, Two volatile beta-chromenes from Wisteria sinensis flowers, Phytochemistry, 1994; 37:17691770.
[11] Nolan KA, Zhao H, Faulder PF, Frenkel AD, Timson DJ, Siegel D, Ross D, Burke Jr T R, Stratford IJ, Bryce RA, Coumarin-based inhibitors of human NAD(P)H: quinone oxidoreductase-1. Identification, structure-activity, off-target effects and in vitro human pancreatic cancer toxicity, Journal of Medicinal Chemistry, 2007; 50:6316-6325.

[12] Doshi JM, Tian D, Xing C, Structure- activity relationship studies of ethyl 2-amino-6-bromo-4-(1-cyano-2-ethoxy-2oxoethyl)-4 H-chromene-3-carboxylate (HA 14-1), an antagonist for antiapoptotic Bcl-2 proteins to overcome drug resistance in cancer, Journal of Medicinal Chemistry, 2006; 49(26):7731-7739.

[13] Kemnitzer W, Drewe J, Jiang S, Zhang H, Crogan-Grundy C, Labreque D, Bubenick M, Attardo G, Denis R, Lamothe S, Gourdeau H, Tseng B, Kasibhatla S, Cai SX, Discovery of 4-Aryl$4 \mathrm{H}$-chromenes as a New Series of Apoptosis Inducers Using a Cell- and Caspase-Based High Throughput Screening Assay. 4. Structure-Activity Relationships of N-Alkyl Substituted Pyrrole Fused at the 7,8-Positions, Journal of Medicinal Chemistry, 2008; 51(3):417-423.

[14] Stolle, T. Szuppa, S. E. S. Leonhardt and B. Ondruschka, Ball milling in organic synthesis: a. solutions and challenges, Chemical Society of Review, 2011; 40: 2317-2329; (b) G. W. Wang, Mechanochemistry of fullerenes and related materials, Chemical Society of Review, 2013; 42: 7668-7700; (c) R. B. Nasir-Baig and R. S. Varma, Stereo- and regio-selective one-pot synthesis of triazole-based unnatural amino acids and $\beta$-amino triazole Journal of communication, 2012;41: 1559 -1584.

[15] J. G. Hernández and E. Juaristi, Asymmetric Aldol Reaction Organocatalyzed by $(S)$ - Proline-Containing Dipeptides: Improved Stereoinduction under Solvent-Free Conditions Journal of Organic. Chemistry .2011; 76: 5, 1464-1467; (b) T Chatterjee, D. Saha and B. C. Ranu, Solvent-free transesterification in a ball-mill over alumina surface, Tetrahedron Letters; 2012: 53, 4142-4144; (c) M. Nüchter, B. Ondruschka, W. Bonrath and A. Gum, Microwave assisted synthesis - a critical technology overview , Journal of Green Chemistry, 2004;6:128-141. (d) J. Mokhtari, M. R. Naimi-Jamal, H. Hamzeali, M. G. Dekamin and G. Kaupp, Kneading ballmilling and stoichiometric melts for the quantitative derivatization of carbonyl compounds with gas-solid recovery, sustainable chemistry,2009;2: 248-254. (e) M. R. Naimi-Jamal J. Mokhtari, M. G. Dekamin and G. Kaupp, Sodium Tetraalkoxyborates: Intermediates for the Quantitative Reduction of Aldehydes and Ketones to Alcohols through Ball Milling with $\mathrm{NaBH}_{4}$ European Journal of Organic Chemistry .,2009, 3567-3572 